\title{
Contents, Vol. 25, 1911
}

Inhalts • Verzeîchnis.

Original-Arbeiten.

Adam, Curt, Statistisch.es, Klinisches und Anatomisches

über das Glioma retinae $\quad 330$

Ask, Fritz, Fall von Entwicklungsanomalie (rinnenförmige

Einsenkung des Lidrandes) im Gebiete des unteren

Tränenröhrchens 178

Axenfeld, Th., Varicenbildung auf der Papille ...... 362

Bergmeister, Rudolf, Ein Beitrag zur Histologie der Stauungs-

papille. (Hierzu Taf. Ill) 49

Burgers, Th. J., Über den Flüssigkeitswechsel des Auges 223 Cords, Richard, Zur Beurteilung der Adrenalinmydriasis . 350 Cramer, E., Über anderweitige Verwendung der Kuhntschen Operation des Blepharitisektropiums 459

Denig, Rudolf, Transplantation bei chronischem tracho-

matösem Pannus

278

Dutoit, A., Ein Beitrag zur Kasuistik der Keratitis neuro-

paralytica 253

- -Traumatische Pneumokokken-Panophthalmie. (Hierzu

Taf. XI) 542

E $1>$ penstein, Arthur, Untersuchungen über den Gehalt der Iris

an elastischen Fasern unter normalen und pathologischen

Verhältnissen. (Hierzu Taf. X) 513

Grunert, Terminol, eine neue Cuprum-citricum Salbe zur

Behandlung von Trachom und Conjunctivitis folli-

cularis 523

Heller, 8., Prophylaktische Maßnahmen für später Er-

blindende

67

Hesse, Rob., Hat die operative Therapie der hochgradigen

Myopie eine Berechtigung? 417

Höeg, Niels, Traunxatische Skleralruptur bei Buphthalmus 191 Junius, Zur Ätiologie des

Trachoms. (Hierzu Taf. IV-V) . 129

$-\mathrm{IV}-$

Kuhnt, H., Wie vermeiden wir Entstellungen bei nm-

schriebenen Abtragungen der Lidränder in ganzer

Dicke 272

Kuschél, J., Zwei Fälle von Unterbrechung des intraokularen

Flüssigkeitsstromes im Bereiche seines eiliaren $\mathrm{Ab}$ -

schnittes nach Kontusion des Augapfels 462

Lindenmeyer, Otto, Die Operation des totalen Lymph- 
blepharon nach Weeks

267

Mügge, Felix, Ein Beitrag zur Leberschen f amiliären Opticus-

atrophie $\quad 236$

Neschitsch, Georg, Beitrag zur Heilung der ektatischen Horn-

hautnarben. Trepanation des Staphyloms 527

Pick, L., Über die Fuchssche Maculaerkrankung bei Myopie 54 Schindler, Otto, Über

Behandlung des Xanthelasma mit

Radiom 62

Schmidt, Heinrich, Der einzügige Starschnitt. (Hierzu

Taf. II) 28, 154

Seef elder, R., Anatomischer Befund in einem Falle von an-

geborener Ektopie der Pupille mit Linsenluxation.

(Hierzu Taf. IX) 353

Siebenlist, Walter., Zur Myopiefrage. (Statistische Unter-

suchungen.) 443

Steiger, Adolf, Sind Hornhauttrübungen eine Ursache der

Myopie?

41

Steindorff, Kurt, Über Barlowsche Krankheit mit besonderer

Berücksichtigung der dabei beobachteten Augen-

erscheinungen 180

Stilling, J., Über den Mechanismus der Akkommodation.

(Hierzu Taf. I) 15, 141

Weiß, Otto, Der intraokulare Flüssigkeitswechsel .... 1 Werncke, Th., UberAugenveränderungen bei der Bubonenpest 186 Wessely, K., Über den intraokularen Flüssigkeitswechsel.

(Hierzu Taf. V-VII) 315

Wirths, M., Beitrag zor Kenntnis der persistierenden Pupillar-

membran. (Hierzu Taf. VIII) 363

Berichte über die deutsche ophthalmologisehe Literatur.

Die bisherigen Erfolge mit Salvarsan in der Augenheilkunde.Sammelreferat, abgeschlossen am

1. I. 1911. Von Dr. .Richard Cords in Bonn 88

$\leftarrow \mathrm{V}-$

Diagnostik und Extraktion von Tremdkörpern. Von Dr.

Asmus in Düsseldorf 469

Untersuchungsmethoden. (I. u. II. Semester 1909.) Von

Prof. Dr. F. Dimmer in Wien 367

Experimentelle Pathologie und pathologische Anatomie 1910.

Von Prof. v. Michel in Berlin 479, 550

Dioptrik und Anomalien der Refraktion und Akkommodation.

(II. Semester 1910.) Referent: Prof. Dr. Maximilian

Salzmann in Wien 372

Anatomie des Auges. (I. Semester 1910.) Von Prof. Dr.

Sobotta in Würzburg 72

Berichte über die ausländische ophthalmologische Literatür.

Bericht über die amerikanische Literatür (1909). Von Prof.

Dr. E. Denig in New York 279

Bericht über die englische ophthalmologische literatür. 
(II. Semester 1909.) Von Dr. Markus in London . . 287 Bericht über die italienische Literatür des Jahres 1909. Von

Dr. Speciale-Cirincione in Rom 376

Bericht über die skandinavische ophthalmologische Literatür.

(I. u. II. Semester 1909.) Von Priv.-Doz. Dr. Ernst

Forsmarh in Stockholm . .'. 82

Bericht über die russische ophthalmologische Literatür.

I. Semester 1910. Von Priv.-Doz. Dr. T-h. Werncke in

Odessa 197

Gesellschaftsberichte.

Verein der Augenärzte von Ost- und Westpreußen

Sitzung am 19. $•$ Febr. 1911 in Königsberg 484

Berliner ophthalmologische Gesellschaft

Sitzung vom 24. November und 15. Dezember 1910 . Ill

Sitzung vom 26. Januar 1911298

Sitzung vom 23. Februar und 9. März $1911 \ldots 400$

Ophthalmologische Gesellschaft in Wien

Sitzung vom 26. Oktober und 16. November 1910 . . 103 Sitzung vom 16. Januar und 20.

Februar 1911 . . 489

Sitzungen der Gesellschaft der Augenärzte in Moskau vom11. Oktober, 8. November, 13.

Dezember 1910, 2. Jamiar.31. Januar und 7. März 1911497

St. Petersburger ophthalmologische Gesellschaft

Sitzung vom 25. November 1910 und 27. Januar 1911300 Sitzungen vom 3. März, 24. März und 21. April 1911.578

$-\mathrm{VI}-$

Kleine Mitteilung 405

ünfall- und Versicherungskunde. . 116, 212, 301, 406, 501, 579

Therapeutisehe Umschau .... 118, 212, 303, 407, 503, 582

Diagnostisehe Notizen $\quad 120,215,306,410,506, \quad 584$

Buehanzeigen 217, 309, 508

Literatur-Verzeichnis $125,219,310,413,509, \quad 588$

Offene Korrespondenz $102,218, \quad 412$

Tagesnachrichten 128, 314

Personalien 128, 222, 314, 416, 591

Albert-Mooren-Denkmal

S. Sachregister

592

Namenregister 